Classification

Physics Abstracts

$05.20-61.40 \mathrm{~K}-64.75-81.20 \mathrm{~S}$

\title{
Model calculations for wetting transitions in polymer mixtures
}

\author{
I. Schmidt and K. Binder \\ Institut für Physik, Johannes-Gutenberg Universität, Postfach 3980, D-6500 Mainz, F.R.G.
}

(Reçu le 13 mai 1985, accepté le 6 juin 1985)

\begin{abstract}
Résumé. - Nous étudions des mélanges binaires partiellement compatibles de polymères flexibles en présence d'une paroi qui adsorbe préférentiellement un des composants. Utilisant une approximation de champ moyen du genre Flory-Huggins, nous montrons que, dans le cas générique, à la coexistence des deux phases, la paroi est toujours " mouillée ", c'est-à-dire recouverte d'une couche d'épaisseur macroscopique de la phase préférée. Nous montrons aussi que, sur la courbe de coexistence, la transition vers l'état non mouillé se passe à des fractions de volume d'ordre $1 / \sqrt{N}$ ( $N$ est la longueur de la chaîne). Nous trouvons à la fois des transitions de mouillage du premier et du second ordres et nous étudions la variation, à travers la transition, de l'épaisseur de la couche de surface, de l'énergie de surface et d'autres quantités reliées. Nous discutons à la fois la validité de l'approximation de grande longueur d'onde que nous utilisons et les effets possibles de fluctuations sur le " mouillage critique ". Nous comparons nos résultats à des simulations numériques de Monte Carlo du mouillage dans des modèles de type Ising. Nous mentionnons brièvement le rapport de nos résultats avec des travaux antérieurs et avec de possibles conséquences expérimentales.
\end{abstract}

\begin{abstract}
Partially compatible binary mixtures of linear flexible polymers are considered in the presence of a wall which preferentially adsorbs one component. Using a Flory-Huggins type mean field approach, it is shown that in typical cases at two-phase coexistence the wall is always " wet ", i.e. coated with a macroscopically thick layer of the preferred phase, and the transition to the non wet state occurs at volume fractions of the order of $1 / \sqrt{N}$ (where $N$ is the chain length) at the coexistence curve. Both first and second order wetting transitions are found, and the variation of the surface layer thickness, surface excess energy and related quantities through the transition is studied. We discuss both the validity of the long wavelength approximation involved in our treatment, and possible fluctuation effects for " critical wetting ", comparing our results to Monte Carlo simulations of wetting in Ising models. The relation of our results to previous work and possible experimental consequences are also briefly mentioned.
\end{abstract}

\section{Introduction.}

A surface of a container enclosing a fluid mixture, which has a miscibility gap and is held at a composition corresponding to one of the coexisting phases, may be completely wetted by the other phase. This phenomenon of " complete wetting " is predicted to occur in all binary mixtures close enough to their critical point of unmixing [1]. Changing the state of the system such that one moves along the coexistence curve to a region of low mutual solubility, one undergoes a "wetting transition" from the wet state, where the surface is coated with a macroscopically thick layer of the phase preferred by the wall, to a non-wet state, where this thickness is microscopically small. This transition typically is first order but may also be second order (" critical wetting " [2-24]), the order of the transition changes at a wetting tricritical point [2-4]; if the wetting transition is first order there should also exist a precursor phenomenon in the one phase region where the thickness of the adsorbed surface layer jumps from a small to a large but finite value (" prewetting " [1]).

While there thus is an enormous theoretical activity on this problem, experimental work so far reports observations of first-order wetting transitions only [25-33]. It does not seem easy to find systems suitable for the observation of critical wetting. In order to understand this problem further it clearly is necessary to look into the details of specific systems more closely. This is one motivation why it should be interesting to study mixtures of linear flexible macromolecules which is the subject of the present work : in polymer mixtures, the degree of polymerization (in our description, the "chain lengths" $N_{\mathrm{A}}, N_{\mathrm{B}}$ of polymer species A, B) can be varied in a controlled fashion over a wide range (for a practical example, see e.g. [34]), while the basic interactions 
between monomers (as well as monomer-wall interactions) should be essentially independent of chain length (apart from end effects). In addition, since the chemical structure of these macromolecules is so complicated, one may well have somewhat different effective interactions than for the case of mixtures of small molecules. Since in (partially) compatible polymer mixtures the effective energy responsible for unmixing, measured as usual by the Flory-Huggins parameter [35] $\chi$, is much smaller than unity [energy being measured in units of $K_{\mathrm{B}} T, T$ being the temperature and $K_{\mathrm{B}}$ Boltzmann's constant], there is no reason to assume that interactions between the monomers and the wall will be similarly small. Consequently, the region where the wall is wet is not restricted to the vicinity of criticality. One thus expects to observe the wetting transition in a region where the mutual solubility of the mixture is small.

Thus one expects drastic surface effects in thin films of mixed polymers. In addition, the growth of the wetting layer into the bulk may be an important mechanism of phase separation, when one studies mixtures of polymers quenched into the two-phase regions, which is a problem of current theoretical [36, 37] and experimental [38] interest. Additional kinetic effects are expected to be associated with the (firstorder) wetting transition itself : in the wet phase, the non-wet state may be metastable until one reaches a " surface spinodal » [23], and vice versa. For mixtures of small molecules the limit of metastability in the bulk (standard "spinodal ») is of little physical significance [39], because of fluctuation effects, and thus it is questionable whether surface spinodals are significant then. For polymer mixtures, on the other hand, the mean field character of the unmixing transition [40] also has the consequence that the bulk spinodal gets a well-defined meaning, for $N_{\mathrm{A}}$, $N_{\text {B }} \rightarrow \infty$ [37]; we also expect that surface spinodals are significant then. In addition, since polymers are rather slow objects one might also more easily follow the decay of metastable surface states [23].

In section 2, we formulate our model and derive some general results, while section 3 gives typical numerical results for the quantities of interest. Our treatment is closely related to that of Nakanishi and Pincus [23], but unlike the latter authors we do not restrict the treatment to the vicinity of the bulk critical unmixing, as it is very unlikely that the wetting transition occurs there; thus we also disagree with their conclusion that critical wetting is nearly impossible for polymer blends. In section 4 , we then discuss fluctuation effects on critical wetting for polymer mixtures, and for the sake of comparison discuss some recent Monte Carlo results on critical wetting in the Ising model [41]. Section 5 summarizes our conclusions.

\section{Phenomenological theory.}

We start by writing down an expression for the bulk free energy of the polymer mixture in the long wavelength approximation [23, 36, 37], for a semi-infinite system $(z>0)$ \{ surface area with surface element $\mathrm{d} A$ located at $z=0\}$

$$
\frac{F_{\mathrm{b}}}{k_{\mathrm{B}} T}=\int \mathrm{d} A \int_{0}^{\infty} \mathrm{d} z\left\{\frac{\Phi}{N_{\mathrm{A}}} \ln \Phi+\frac{1-\Phi}{N_{\mathrm{B}}} \ln (1-\Phi)+\chi \Phi(1-\Phi)-\Delta \mu \Phi+\frac{a^{2}}{36 \Phi(1-\Phi)}(\nabla \Phi)^{2}\right\}
$$

Equation (1) is based on considering a simple cubic lattice of spacing $a$, each cell of which is either taken by one effective unit of $\mathrm{A}$ (there are $N_{\mathrm{A}}$ such units along an $A$ chain) or $B, \Phi$ is the volume fraction of $A$, and $\Delta \mu$ the chemical potential difference (for convenience we work in the grandcanonical ensemble although in practice $\Phi$ rather than $\Delta \mu$ is the experimentally controlled parameter). The interaction parameter $\chi$ (leading to unmixing for $\chi>0$ ) is found to depend on temperature $T$ and volume fraction $\Phi$ (and even on chain length if end effects are included) [42], but we shall ignore the dependence of $\chi$ on $\Phi$ and $N_{\mathrm{A}}, N_{\mathrm{B}}$ in the following, and also assume the polymers to be monodisperse. More accurate theories of polymer mixtures avoiding this Flory-Huggins approach [35] are possible [43], but are rather complicated and involve many parameters, and hence will not be considered here.

The lattice spacing $a$ of the Flory-Huggins lattice can be related to the effective Kuhn step lengths $\sigma_{\mathrm{A}}, \sigma_{\mathrm{B}}$ of the chains (defined from gyration radii $\left.R_{\mathrm{gyr}}^{\mathrm{A}}=\sigma_{\mathrm{A}} \sqrt{N_{\mathrm{A}} / 6}, R_{\mathrm{gyr}}^{\mathrm{B}}=\sigma_{\mathrm{B}} \sqrt{N_{\mathrm{B}} / 6}\right)$ as $[36,37,44]$

$$
a^{2}=\sigma_{\mathrm{A}}^{2}(1-\Phi)+\sigma_{\mathrm{B}}^{2} \Phi ;
$$

however, in the following we shall simplify the problem by implying complete symmetry $N_{\mathrm{A}}=N_{\mathrm{B}}, \sigma_{\mathrm{A}}=\sigma_{\mathrm{B}}$ ( $=a$, independent of $\Phi$ ), deferring a discussion of asymmetry effects to the end of this section. It is also important to recall that even under the chosen assumptions equation (1) is quantitatively reliable only in the long wavelength limit

$$
a^{2}(\nabla \Phi)^{2} \ll N_{\mathrm{A}}^{-1}, \quad a^{2}(\nabla \Phi)^{2} \ll N_{\mathrm{B}}^{-1} ;
$$

however, as will be discussed below (see also [45]), equation (1) provides a qualitatively reasonable description for the present problem also when equa- 
tion (3) is not fulfilled. In order to show this we shall treat the limit $N_{\mathrm{A}}, N_{\mathrm{B}} \rightarrow \infty$ by the method of Helfand and coworkers $[46,47]$ for the study of interfacial properties [equation (1) corresponds to the treatment plete treatment applying the numerical techniques of Noolandi and Hong [49] is left to future work.

The perturbing effect of the surface is described by an additional contribution, the "bare " surface free energy $F_{\mathrm{s}}^{(b)}$, which is assumed to depend on the local volume fraction $\Phi_{1} \equiv \Phi(z=0)$ at the surface only

$$
\begin{aligned}
F_{\mathrm{s}}^{(\mathbf{b})} / k_{\mathrm{B}} T & =\int \mathrm{d} A \int_{-\infty}^{+\infty} \mathrm{d} z f_{\mathrm{s}}^{(\mathbf{b})}(\Phi, z) \\
& =\int \mathrm{d} A \int_{-\infty}^{+\infty} \mathrm{d} z f_{\mathrm{s}}^{(\mathrm{b})}(\Phi) \delta(z)=A f_{\mathrm{s}}^{(\mathbf{b})}\left(\Phi_{1}\right)
\end{aligned}
$$

A simple explicit expression for $f_{\mathrm{s}}^{(\mathbf{b})}$ is obtained using linear and quadratic terms in $\Phi$, analoguous to equation (1), if $\Phi_{1}$ is small, and again omitting unimportant constant terms of polymer interfaces in reference [48]]. A more com-

$$
f_{\mathrm{s}}^{(\mathrm{b})}\left(\Phi_{1}\right)=-\mu_{1} \Phi_{1}-\frac{1}{2} g \Phi_{1}^{2}, \quad\left(\mu_{1}+g / 2>0\right)
$$

where $\mu_{1}$ plays the role of a chemical potential difference favouring species $A$ in the surface layer, and $g$ represents the change of interactions near the surface (including the effects due to "missing neighbours", etc.). Hence $g$ can be positive as well as negative. We shall discuss the consequences of choosing a more general form for $f_{\mathrm{s}}^{(\mathrm{b})}$ occasionally, and will also briefly comment on long-range surface perturbations where $f_{\mathrm{s}}^{(\mathrm{b})}(\Phi, z)$ decays towards zero for $z \rightarrow \infty$ in power-law form, as it happens for van der Waals forces between the surface and the molecules in the mixture [16-18]. If instead $1-\Phi_{1} \ll 1$, we may expand $f_{\mathrm{s}}^{(\mathrm{b})}=-\mu_{1}^{\prime}\left(1-\Phi_{1}\right)-\frac{1}{2} g\left(1-\Phi_{1}\right)^{2}$, which apart from an unimportant constant again reduces to equation (5).

As usual we disregard any inhomogeneities in directions parallel to the surface, and find the equilibrium solution from minimizing the total free energy $\{A$ is the total surface area $\}$

where

$$
\frac{\delta}{\delta \Phi}\left[\frac{F_{\mathrm{b}}+F_{\mathrm{s}}^{(\mathrm{b})}}{A k_{\mathrm{B}}^{-} T}\right]=0
$$

$$
\begin{aligned}
\frac{F_{\mathrm{b}}+F_{\mathrm{s}}^{(\mathrm{b})}}{A k_{\mathrm{B}}^{-} T}=\int_{0}^{\infty} \mathrm{d} z\left\{\frac{1}{N}[\Phi \ln \Phi+(1-\Phi) \ln (1-\Phi)]+\right. & \chi \Phi(1-\Phi)-\Delta \mu \Phi+ \\
& \left.+\frac{a^{2}}{36 \Phi(1-\Phi)}\left(\frac{\mathrm{d} \Phi}{\mathrm{d} z}\right)^{2}\right\}-\mu_{1} \Phi_{1}-\frac{1}{2} g \Phi_{1}^{2}
\end{aligned}
$$

The bulk solution $\Phi_{\infty} \equiv \Phi(z \rightarrow \infty)$ is found from equations (6), (7) disregarding all surface and gradient terms, and hence described by the equation

$$
\chi=\chi\left(\Phi_{\infty}, \Delta \mu\right)=\frac{1}{N} \frac{\ln \left[\left(1-\Phi_{\infty}\right) / \Phi_{\infty}\right]+N \Delta \mu}{1-2 \Phi_{\infty}} .
$$

At the coexistence curve $\Delta \mu=0$ in this symmetric case. By the notation $\chi\left(\Phi_{\infty}, \Delta \mu\right)$ we have already indicated that it is more convenient to use $\Phi_{\infty}$ and $\Delta \mu$ as independent variables rather than $\chi$ and $\Delta \mu$.

The concentration profile near the surface is now obtained as the solution of the following equation [" phase portrait » $[2,3]]$

$$
\left.\frac{a^{2}}{36 \Phi(1-\Phi)}\left(\frac{\mathrm{d} \Phi}{\mathrm{d} z^{\prime}}\right)^{2}\right|_{z^{\prime}=0} ^{z^{\prime}=z}=\left.\left\{\frac{1}{N}[\Phi \ln \Phi+(1-\Phi) \ln (1-\Phi)]+\chi \Phi(1-\Phi)-\Delta \mu \Phi\right\}\right|_{\Phi_{1}} ^{\Phi(z)} .
$$

Following standard treatments [1-3] the surface excess free energy $F_{\mathrm{s}}$ is then obtained as

$$
F_{\mathrm{s}} /\left(A k_{\mathrm{B}} T\right)=\int_{\Phi_{1}}^{\Phi_{\infty}} \mathrm{d} \Phi\left\{\frac{a^{2}}{18 \Phi(1-\Phi)} \frac{\mathrm{d} \Phi}{\mathrm{d} z}+\mu_{1}+g \Phi\right\}-\mu_{1} \Phi_{\infty}-\frac{1}{2} g \Phi_{\infty}
$$

Minimizing this result with respect to $\Phi_{1}$ yields a boundary condition at the surface,

$$
\left.\frac{a^{2}}{18 \Phi_{1}\left(1-\Phi_{1}\right)} \frac{\mathrm{d} \Phi}{\mathrm{d} z}\right|_{z=0}=-\mu_{1}-g \Phi_{1}=\left(\frac{\partial f_{\mathrm{s}}^{(\mathrm{b})}}{\partial \Phi_{1}}\right) \text {. }
$$


Using this equation together with equation (9) for $z=0$, one finds an explicit solution for $\Phi_{1}$,

$$
\frac{-\partial f_{s}^{(b)}}{\partial \Phi_{1}}=\mu_{1}+g \Phi_{1}=\mp f_{1}\left(\Phi_{1}\right),
$$

with

$$
f_{1}\left(\Phi_{1}\right)=\left[\frac{a}{3} \frac{G\left(\Phi_{1}, \chi\right)-G\left(\Phi_{\infty}, \chi\right)-\Delta \mu\left(\Phi_{1}-\Phi_{\infty}\right)}{\Phi_{1}\left(1-\Phi_{1}\right)}\right]^{1 / 2},
$$

and where $G$ is the free enthalpy per site

$$
G(\Phi, \chi)=N^{-1}[\Phi \ln \Phi+(1-\Phi) \ln (1-\Phi)+\chi \Phi(1-\Phi)],
$$

and the solution yielding the minimum value of $F_{\mathrm{s}}$ has to be taken. Equation (10) can be re-written as

$$
F_{\mathrm{s}} /\left(A k_{\mathrm{B}} T\right)=\int_{\Phi_{1}}^{\Phi_{\infty}} \mathrm{d} \Phi\left[f_{1}(\Phi)-\partial f_{\mathrm{s}}^{(\mathrm{b})}(\Phi) / \partial \Phi\right]
$$

Next we define a local « surface layer susceptibility " $\chi_{1,1}$ as $[50]$

$$
\left.\chi_{1,1} \equiv \frac{\partial \Phi_{1}}{\partial \mu_{1}}\right|_{\Phi_{\infty}, \Delta \mu}=\left\{\left.\frac{\partial}{\partial \Phi_{1}}\left[-f_{1}\left(\Phi_{1}\right)+\partial f_{\mathbf{s}}^{(b)}\left(\Phi_{1}\right) / \partial \Phi_{1}\right]\right|_{\Phi_{\infty}, \Delta \mu}\right\}^{-1}
$$

and similarly

$$
\left.\chi_{1} \equiv \frac{\partial \Phi_{1}}{\partial(N \Delta \mu)}\right|_{\Phi_{\infty}, \mu_{1}}=\left.\chi_{1,1} \frac{\partial}{\partial(N \Delta \mu)}\left[f_{1}\left(\Phi_{1}\right)-\partial f_{\mathbf{s}}^{(b)} / \partial \Phi_{1}\right]\right|_{\Phi_{\infty}, \mu_{1}} .
$$

Keeping $\Phi_{\infty}$ and $\Delta \mu$ fixed in equation (15) means that the Flory-Huggins-Parameter $\chi$ is kept constant [Eq. (8)], and hence this corresponds to a derivative at constant temperature as usual [50], since $\chi$ is controlled by the temperature. Using equation (12) for $f_{1}$ and equation (5) for $f_{\mathbf{s}}^{\text {(b) }}$ it is straightforward to derive explicit expressions for $\chi_{1}$ and $\chi_{1,1}$. These quantities are of particular interest, since the prewetting critical point can be found from the condition $\chi_{1,1}^{-1}=0$; if this occurs at the coexistence curve $(\Delta \mu=0)$ we have a wetting tricritical point. One can also ask how $\Phi_{1}$ responds to a change of temperature (and hence change of $\Phi_{\infty}$ with other parameters staying constant),

$$
\left.\frac{\partial \Phi_{1}}{\partial \Phi_{\infty}}\right|_{\mu_{1} \Delta \mu}=\left.\chi_{11} \frac{\partial}{\partial \Phi_{\infty}}\left[f_{1}\left(\Phi_{1}\right)-\partial f_{\mathbf{s}}^{(\mathbf{b})} / \partial \Phi_{1}\right]\right|_{\mu_{1} \Delta \mu} .
$$

A further quantity of interest is the excess internal energy due to the surface, which becomes,

$$
\begin{gathered}
\left.\frac{U_{\mathrm{s}}}{A k_{\mathrm{B}} T} \equiv \frac{\partial}{\partial(1 / T)}\left(\frac{F_{\mathrm{s}}}{A k_{\mathrm{B}} T}\right)\right|_{\mu_{1}, \Delta \mu}=\left.\frac{\partial \Phi_{\infty}}{\partial(1 / T)} \frac{\partial}{\partial \Phi_{\infty}} \frac{F_{\mathrm{s}}}{A k_{\mathrm{B}} T}\right|_{\mu_{1}, \Delta \mu} \\
\frac{U_{\mathrm{s}}}{A k_{\mathrm{B}} T}=\frac{\partial \Phi_{\infty}}{\partial(1 / T)} \frac{a}{3 N} \int_{\Phi_{1}}^{\Phi_{\infty}} \mathrm{d} \Phi \frac{\Phi(1-\Phi)-\Phi_{\infty}\left(1-\Phi_{\infty}\right)+N \Delta \mu}{\sqrt{\Phi(1-\Phi)\left[G(\Phi, \chi)-G\left(\Phi_{\infty}, \chi\right)-\Delta \mu\left(\Phi-\Phi_{\infty}\right)\right.}} .
\end{gathered}
$$

Finally we consider the solution for the concentration profile itself, which results from equation (9) as

$$
\frac{6 z}{a}=\int_{\Phi_{1}}^{\Phi(z)} \mathrm{d} \Phi /\left\{\Phi(1-\Phi)\left[G(\Phi, \chi)-G\left(\Phi_{\infty}, \chi\right)-\Delta \mu\left(\Phi-\Phi_{\infty}\right)\right]\right\}^{1 / 2} .
$$

This expression shows that the profile is exclusively determined by the bulk properties of the mixture the boundary condition at the surface enters only through the lower integration limit : one thus finds just the same type of profile which would exist also between the bulk co-existing phases $\Phi_{\text {coex }}^{(1)}=\Phi_{\infty}$, $\Phi_{\text {coex }}^{(2)}=1-\Phi_{\infty}$; the distinction only is that the surface can cut off this interfacial profile at any value $\Phi_{1}$ intermediate between $\Phi_{\infty}$ and $1-\Phi_{\infty}$. Of course, if $\Phi_{1} \rightarrow 1-\Phi_{\infty}$ in equation (19) $z \rightarrow \infty$. Thus, if $\Phi_{1}>1-\Phi_{\infty}$ (we assume $\Phi_{\infty}<\Phi_{\text {crit }}=1 / 2$, so that we assume an A-rich phase in the container), the profile decreases first from $\Phi_{1}$ to $1-\Phi_{\infty}$ as $z \rightarrow \infty$, while the interface from this B-rich layer at the sur- 
face to the A-rich phase in the bulk is at a macroscopic distance. This is the description of the "wet " state of the surface in this framework.

An interesting quantity hence is the thickness $D$ of the B-rich layer which we define as

$$
D=\left|-\int_{0}^{\infty} \frac{\Phi(z)-\Phi_{\infty}}{1-2 \Phi_{\infty}} \mathrm{d} z\right| .
$$

For $\Phi$ near $\Phi_{\infty}$ we have [45]

$$
\Phi(z)-\Phi_{\infty} \propto \exp \left(-z / \xi_{\text {coex }}\right),
$$

where the correlation length of concentration fluctuations at the coexistence curve is given by [37]

$$
\begin{aligned}
\xi_{\text {coex }}= & \frac{a}{6} \sqrt{2 N} \times \\
& \times\left\{1-\frac{2 \Phi_{\infty}\left(1-\Phi_{\infty}\right) \ln \left(\left(1-\Phi_{\infty}\right) / \Phi_{\infty}\right)}{1-2 \Phi_{\infty}}\right\}^{-1 / 2}
\end{aligned}
$$

$D$ is only meaningful (finite) in the non-wet state, where $\Phi_{1}<1-\Phi_{\infty}$. A second order wetting transition hence is obtained, when upon change of $\Phi_{\infty}$ for $\Delta \mu=0$ the surface concentration $\Phi_{1}$ smoothly moves towards the critical value

$$
\Phi_{1}^{\text {crit }}=\Phi_{\text {coex }}^{(2)}\left(=1-\Phi_{\infty}\right)\{\text { critical wetting }\} .
$$

From equations (15), (23) it is easy to show that the susceptibility $\chi_{11}$ stays finite at the critical wetting transition. In fact, $\chi_{11}$ has a jump singularity there, it jumps from the value $\chi_{11}^{+}$to a value $\chi_{11}^{-}$, where

$$
\chi_{11}^{ \pm}=\left[\frac{\partial^{2} f_{\mathrm{s}}^{(\mathrm{b})}}{\partial \Phi_{1}^{2}} \mp \frac{a}{6} \sqrt{\frac{2 G^{\prime \prime}\left(\Phi_{\infty}, \chi\right)}{\Phi_{\infty}\left(1-\Phi_{\infty}\right)}}\right]^{-1}
$$

where $G^{\prime \prime}(\Phi, \chi)=\partial^{2} G(\Phi, \chi) / \partial \Phi^{2}$. Also the slope of $\chi_{11}$ stays finite as $\Phi_{1}$ tends to the critical value $\Phi_{1}^{\text {crit }}=$ $1-\Phi_{\infty}$. We note

$$
\left.\frac{\partial \chi_{11}}{\partial \mu_{1}}\right|_{\Phi_{\infty}, \Delta \mu}=\left.\chi_{11} \frac{\partial \chi_{11}}{\partial \Phi_{1}}\right|_{\Phi_{\infty}, \Delta \mu}
$$

and find for $\Phi_{1}=\lim _{\varepsilon \rightarrow 0^{+}}\left(1-\Phi_{\infty} \mp \varepsilon\right)$ that (putting now $\frac{\partial^{2} f_{\mathrm{s}}^{(\mathrm{b})}}{\partial \Phi_{1}^{2}}=g$ for simplicity)

$$
\left.\frac{\partial \chi_{11}}{\partial \Phi_{1}}\right|_{\Phi_{\infty}, \Delta \mu, \Phi_{1}=1-\Phi_{\infty}}=\frac{a}{6} \frac{\left(1-2 \Phi_{\infty}\right) \sqrt{2 G^{\prime \prime}\left(1-\Phi_{\infty}, \chi\right)}}{\sqrt{\Phi_{\infty}\left(1-\Phi_{\infty}\right)}}\left\{g \sqrt{\Phi_{\infty}\left(1-\Phi_{\infty}\right)} \pm \frac{a}{6} \sqrt{2 G^{\prime \prime}\left(1-\Phi_{\infty}, \chi\right)}\right\}^{-2} .
$$

One also finds that the jump $\chi_{11}^{+}-\chi_{11}^{-}$vanishes when $N \rightarrow \infty$ at fixed $\Phi_{\infty}$.

In order to check where critical wetting occurs and where first order wetting occurs, we locate the wetting tricritical point, for which equation (23) must hold and in addition $\chi_{1,1}^{-1}=0$. This yields

$$
\left.\left\{\frac{\partial}{\partial \Phi_{1}}\left[-f_{1}\left(\Phi_{1}\right)+\partial f_{\mathrm{s}}^{(\mathbf{b})} / \partial \Phi_{1}\right]\right\}\right|_{\Phi_{\infty}, \Delta \mu=0, \Phi_{1}=1-\Phi_{\infty}}=0 .
$$

From equation (24) one can check that only $\chi_{11}^{+}$(and $\partial \chi_{11}^{+} / \partial \Phi_{1}$ ) diverge at the tricritical point, while $\chi_{11}^{-}$and the associate slope stay finite. Since the value of the Flory-Huggins parameter $\chi$ at the critical unmixing tempperature is $\chi_{\mathrm{c}}=2 / N$, we conclude that for the function $G$ (Eq. (13)) is of order $N^{-1}$, and hence the function $f_{1}$ (Eq. (12)) is of order $N^{-1 / 2}$. As a result, we conclude from equation (25a)

$$
\left.\frac{\partial^{2} f_{\mathrm{s}}^{(\mathrm{b})}}{\partial \Phi_{1}^{2}}\right|_{\Phi_{\infty}, \Delta \mu=0, \Phi_{1}=1-\Phi_{\infty}}=N^{-1 / 2} f_{2}\left(\Phi_{\infty}\right) / \Phi_{\infty},
$$

where the function $f_{2}\left(\Phi_{\infty}\right)$ is finite for $\Phi_{\infty} \rightarrow 0$ and using equations (12), (13) becomes

$$
f_{2}\left(\Phi_{\infty}\right)=\frac{a}{\sqrt{18}\left(1-\Phi_{\infty}\right)}\left[1+2 \frac{\Phi_{\infty}\left(1-\Phi_{\infty}\right)}{1-2 \Phi_{\infty}} \ln \frac{\Phi_{\infty}}{1-\Phi_{\infty}}\right]^{1 / 2} .
$$

Thus equation (25) can be satisfied only if $-\partial^{2} f_{\mathrm{s}}^{(\mathrm{b})} /\left.\partial \Phi_{1}^{1}\right|_{\Phi_{\infty}, \Delta \mu=0, \Phi_{1}=1-\Phi_{\infty}}$ (which is simply the constant $g$ if one uses Eq. (5)) is negative. Since this quantity typically will be of order unity, we can satisfy equation (25) only for $\Phi_{\infty} \propto N^{-1 / 2}$, and hence $f_{2}\left(\Phi_{\infty}\right) \approx a / \sqrt{18}$ then, and hence we find the tricritical wetting point for

$$
\left(\Phi_{\infty}\right)_{\text {tricrit }} \approx \frac{a}{\sqrt{18 N}} /\left.\frac{\partial^{2} f_{\mathrm{s}}^{(\mathrm{b})}}{\partial \Phi_{1}^{2}}\right|_{\Phi_{\infty}, \Delta \mu=0, \Phi_{1}=1-\Phi_{\infty}}\left(=-\frac{a / g}{\sqrt{18 N}}\right) .
$$


The generalization of this result to the asymmetric case $\left(\sigma_{\mathrm{A}} \neq \sigma_{\mathrm{B}}, N_{\mathrm{A}} \neq N_{\mathrm{B}}\right)$ in the limit $N_{\mathrm{A}} \rightarrow \infty$ simply is ( $\Phi_{\text {coex }}^{(2)}$ is the concentration at the A-rich branch of the coexistence curve)

$$
\left(\Phi_{\infty}\right)_{\text {tricrit }} \approx \frac{\sigma B}{\sqrt{18 N_{\mathrm{A}}}} /\left.\frac{\partial^{2} f_{\mathrm{s}}^{(\mathrm{b})}}{\partial \Phi_{1}^{2}}\right|_{\Phi_{\infty}, \Delta \mu=\Delta \mu_{\mathrm{coex}}, \Phi_{1}=\Phi_{\text {coex }}^{(2)}}
$$

From the general topology of the wetting phasediagrams [4] in the space of the variables $\mu, \Delta \mu$, and $T$ (or equivalently, $\Phi_{\infty}$ ) it then immediately follows that for $g>0$ we have always first-order wetting, while for $g<0$ the region of first order wetting occurs for $\Phi_{\infty}<\left(\Phi_{\infty}\right)_{\text {tricrit }}$, while for $\left(\Phi_{\infty}\right)_{\text {tricrit }}<\Phi_{\infty}<\Phi_{\text {crit }}$ $(=1 / 2$ in the symmetric case) critical wetting occurs.

Since $\left(\Phi_{\infty}\right)_{\text {tricrit }}$ is so small (Eqs. (27), (28)), polymer mixtures hence would be excellent candidates of systems to observe critical wetting (at least if the framework of short-range surface perturbations, Eq. (4), would apply). This conclusion is opposite to the work of Nakanishi and Pincus [23]; in our opinion, this discrepancy is due to the fact that their parameter $g$ (which should not be confused with our $g$ ) is of order $N$ for polymer blends, and not of order unity as assumed. Then tricritical wetting does not occur very close to $\Phi_{\text {crit }}$, as they imply, but rather close to $\Phi_{\infty}=0$.

For critical and tricritical wetting the condition that $\Phi_{1}=\Phi_{\text {coex }}^{(2)}\left(=1-\Phi_{\infty}\right.$ for symmetric mixtures) also implies that the profile at $z=0$ is flat, the interface from $\Phi_{\text {coex }}^{(2)}$ at the surface to $\Phi_{\text {coex }}^{(1)}=\Phi_{\infty}$ is infinitely far apart, and hence $\mathrm{d} \Phi / \mathrm{d} z=0$ at the surface. From equation (11) we then conclude that critical wetting occurs for

$$
0=\partial f_{\mathrm{s}}^{(\mathrm{b})} /\left.\partial \Phi_{1}\right|_{\Phi_{1}=\Phi_{\text {coex }}^{(2)}}
$$

or

$$
0=-\mu_{1}-g\left(1-\Phi_{\infty}\right), \quad \mu_{1}^{\mathrm{c}}=-g\left(1-\Phi_{\infty}\right)
$$

Hence tricritical wetting is only possible for

$$
\left(\mu_{1}^{\mathfrak{c}}\right)_{\text {tricrit }}=-g\left\{1-\left(\Phi_{\infty}\right)_{\text {tricrit }}\right\} \approx-g+\frac{a}{\sqrt{18 N}} .
$$

If $\Phi_{\infty}>\left(\Phi_{\infty}\right)_{\text {tricrit }}$ we have the surface nonwet for $\mu_{1}<\mu_{1}^{\mathrm{c}}(D<\infty)$ and wet for $\mu_{1}>\mu_{1}^{\mathrm{c}}(D=\infty)$. If for fixed $\mu_{1}<\left(\mu_{1}^{\mathfrak{c}}\right)_{\text {tricrit }}$ we vary $\Phi_{\infty}$ (by changing the temperature, following the coexistence curve), we obtain the critical wetting transition at (we must have $-1<\frac{\mu_{1}}{g}<-1 / 2$ in order to have wetting at the A-rich side at all)

$$
\Phi_{\infty}^{\text {crit }}=1+\mu_{1} / g .
$$

In the case of a more general choice of $f_{\mathbf{s}}^{(\mathbf{b})}$ than equation (5) we find $\Phi_{\infty}^{\text {crit }}$ from the solution of equation (29a).

A fully explicit treatment is also possible if $\sigma_{\mathrm{A}} \neq \sigma_{\mathrm{B}}$ but still $N_{\mathrm{A}}=N_{\mathrm{B}}=N$. Then equations (25), (26) yielding the wetting tricritical point is replaced by

$$
\left.\frac{\partial^{2} f_{\mathrm{s}}^{(\mathrm{b})}}{\partial \Phi_{1}^{2}}\right|_{\Phi_{\infty}, \Delta \mu=0, \Phi_{1}=1-\Phi_{\infty}}=\frac{1}{\sqrt{18}} \sqrt{\sigma_{\mathrm{B}}^{2}+\left(\sigma_{\mathrm{A}}^{2}-\sigma_{\mathrm{B}}^{2}\right) \Phi_{\infty}} \sqrt{\frac{\partial^{2} G\left(\Phi_{\infty}, \chi\right)}{\partial \Phi_{\infty}^{2}} / \Phi_{\infty}\left(1-\Phi_{\infty}\right)}
$$

If $\sigma_{\mathrm{B}}^{2} / \sigma_{\mathrm{A}}^{2} \ll \Phi_{\infty}$ equation (32) leads to $\left(\Phi_{\infty}\right)_{\text {tricrit }} \approx\left(\frac{\sigma_{\mathrm{A}}}{\sqrt{18 N}} /\left.\frac{\partial^{2} f_{\mathrm{s}}^{(b)}}{\partial \Phi_{1}^{2}}\right|_{\Phi_{\infty}, \Delta \mu=0, \Phi_{1}=1-\Phi_{\infty}}\right)^{2}$ instead of equation (28), i.e. there is an intermediate regime where $\left(\Phi_{\infty}\right)_{\text {tricrit }} \propto N^{-1}$ before one crosses over to the asymptotic behaviour $\left(\Phi_{\infty}\right)_{\text {tricrit }} \propto N^{-1 / 2}$ for $N \rightarrow \infty$.

From equation (31) we see that the wetting transition can occur anywhere along the coexistence curve of the polymer mixture, if $-1<\frac{\mu_{1}}{g}<-1 / 2:$ if $\mu_{1} / g>-1 / 2$, the surface stays always non-wet, if $\mu_{1} / g<-1$ it stays always wet (for $N \rightarrow \infty$ ). However, if $\Phi_{\infty}^{\text {crit }}$ is very small $\{$ or if we study the first-order wetting transition possible for $\left.\Phi_{\infty}<\left(\Phi_{\infty}\right)_{\text {tricrit }}\right\}$, the long wavelength theory is inaccurate, because the interfacial profile then becomes very steep in its centre, and an alternative approach is required [46, 47, 49]. Defining a length $L$ from the maximum slope of the profile [45]

$$
L^{-1} \equiv 2\left(\Phi_{\text {coex }}^{(2)}-\Phi_{\text {coex }}^{(1)}\right)^{-1}\left|\frac{\mathrm{d} \Phi}{\mathrm{d} z}\right|_{\max }=2\left(1-2 \Phi_{\infty}\right)^{-1}\left|\frac{\mathrm{d} \Phi}{\mathrm{d} z}\right|_{\Phi=\frac{1}{2}}
$$


one would find from equation (9), putting $\Phi_{1}=\Phi_{\infty}, \Delta \mu=0$,

$$
L^{-1}=6 a^{-1}\left(1-2 \Phi_{\infty}\right)^{-1}\left\{\frac{1}{N}\left[\ln 2-\Phi_{\infty} \ln \Phi_{\infty}-\left(1-\Phi_{\infty}\right) \ln \left(1-\Phi_{\infty}\right)\right]+\chi\left[\frac{1}{4}-\Phi_{\infty}\left(1-\Phi_{\infty}\right)\right]\right\}^{1 / 2}
$$

which tends to $L=(a / 3) \chi^{-1 / 2}$ for $\Phi_{\infty} \rightarrow 0$ [45]. This result implies that in the centre of the profile equation (3) is not fulfilled, and hence it is no surprise that the prefactor $a / 3$ of the last relation is not corroborated by the exact treatment. [46, 47, 49], although the variation with $\chi^{-1 / 2}$ is predicted correctly.

For a treatment of the limit $\Phi_{\infty} \rightarrow 0$ we apply the approach of Helfand and co-workers [46, 47]. Rather than solving a differential equation for $\Phi$ one has to solve two differential equations for the auxiliary functions $q_{\mathrm{A}}(z), q_{\mathrm{B}}(z)$

$$
\begin{aligned}
& \frac{a^{2}}{6} \frac{\mathrm{d}^{2} q_{\mathrm{A}}}{\mathrm{d} z^{2}}=\chi q_{\mathrm{B}}^{4} q_{\mathrm{A}}+\chi q_{\mathrm{A}} \zeta, \\
& \frac{a^{2}}{6} \frac{\mathrm{d}^{2} q_{\mathrm{B}}}{\mathrm{d} z^{2}}=\chi q_{\mathrm{A}}^{4} q_{\mathrm{B}}+\chi q_{\mathrm{B}} \zeta,
\end{aligned}
$$

where $\Phi(z)=q_{A}^{2}(z)$ and the Lagrange multiplyer $\zeta$ ensures the condition $q_{\mathrm{A}}^{2}(z)+q_{\mathrm{B}}^{2}(z) \equiv 1$. These equations have been used for the computation of interfacial profiles and free energies but their derivation by the method of Hong and Noolandi [49] shows that the same equations result also for a problem with a local free surface perturbation; in the limit $N \rightarrow \infty$ the free surface acts as a boundary condition for equation (34) only [51]. Following Helfand [46, 47] we note that equation (34) can be rearranged to yield a « conservation law " for the " Hamiltonian " $\mathfrak{H}$,

$$
\begin{gathered}
\mathcal{H}=\frac{a^{2}}{12}\left[\left(\frac{\mathrm{d} q_{\mathrm{A}}}{\mathrm{d} z}\right)^{2}+\left(\frac{\mathrm{d} q_{\mathrm{B}}}{\mathrm{d} z}\right)^{2}\right]-\frac{1}{2} \chi q_{\mathrm{A}}^{2} q_{\mathrm{B}}^{2} \\
\mathrm{~d} \mathcal{H} / \mathrm{d} z=0
\end{gathered}
$$

Since the minimum free energy in the bulk is obtained for $\mathfrak{H}=0$, equation (35) yields a boundary condition

at the surface $\left[\right.$ note $\left(\frac{\mathrm{d} q_{\mathrm{A}}}{\mathrm{d} z}\right)^{2}=\frac{1}{4 \Phi}\left(\frac{\mathrm{d} \Phi}{\mathrm{d} z}\right)^{2}, \frac{1}{4(1-\Phi)}$ $\left.\left(\frac{\mathrm{d} \Phi}{\mathrm{d} z}\right)^{2}\right]$

$$
\left.\frac{a^{2}}{12}\left(\frac{\mathrm{d} \Phi}{\mathrm{d} z}\right)^{2}\right|_{z=0}=2 \chi \Phi_{1}^{2}\left(1-\Phi_{1}\right)^{2} .
$$

Thus specifying the value $\Phi_{1}$ at the surface uniquely determines the concentration profile. The solution to this problem hence is simply the interfacial profile obtained by Helfand et al. [46, 47],

$$
\Phi(z)=\frac{1}{2}\left[1-\tanh \left(\frac{z-d}{L}\right)\right], \quad L=\frac{a}{\sqrt{6}} \chi^{-1 / 2}
$$

where the inflection point of the profile occurs at a distance $d$ from the surface which follows from

$$
\begin{aligned}
& \Phi_{1}=\frac{1}{2}[1+\tanh (d / L)], \\
& d=-\frac{1}{2} L \ln \left(\Phi_{1}^{-1}-1\right) .
\end{aligned}
$$

A second-order wetting transition hence can occur for $\Phi_{1}=1$ only $(d \rightarrow \infty)$. If $\Phi_{1}<1$ equations (37), (38) describe a non-wet profile. Depending on the behaviour of $f_{\mathrm{s}}^{(\mathrm{b})}\left(\Phi_{1}\right)$, one can again have also a firstorder transition at a point where $F_{\mathrm{s}} /\left(A K_{\mathrm{B}} T\right)$ for the non-wet state becomes equal to $f_{\mathrm{s}}^{(\mathrm{b})}(1)+\sigma, \sigma$ being the interfacial free energy (in units of temperature) between the bulk coexisting phases with concentrations $\Phi=0, \Phi=1$ as obtained in reference [46]. Since equations (20), (37) imply that for $\Phi_{1} \rightarrow 1$ we have $D \approx d$, equation (38) again shows the logarithmic divergence of the thickness of the wet layer when a second-order wetting transition is approached. As a result, we have found that the qualitative features of the description of wetting at $\Phi_{\infty}=0$ in the framework of the long wave length approximation and in the framework of the theory of Helfand et al. [46, 47, 49] are the same, although prefactors (such as the length $L$ in Eqs. (37), (38) and in Eq. (33)) differ.

\section{Numerical results.}

In the framework of the long-wavelength approximation, the problem of obtaining the concentration profile is reduced to a simple quadrature (Eq. (19)). In the regime where the wetting transition is first order, one must obtain $F_{\mathrm{s}}$ from equation (10) through another quadrature both for the wet and the non-wet state, and locate the transition from the intersection of the two surface free energy branches. The thickness $D$, of the layer follows from another quadrature (Eq. (20)). We have done this for a number of typical cases which are described below. We have chosen units such that $a \equiv 1$ for simplicity.

Figure 1 shows typical profiles resulting from equation (19). The dependence on chain length can be completely absorbed in the normalization of the abscissa, and the only relevant parameter is $\Phi_{\infty}$ 

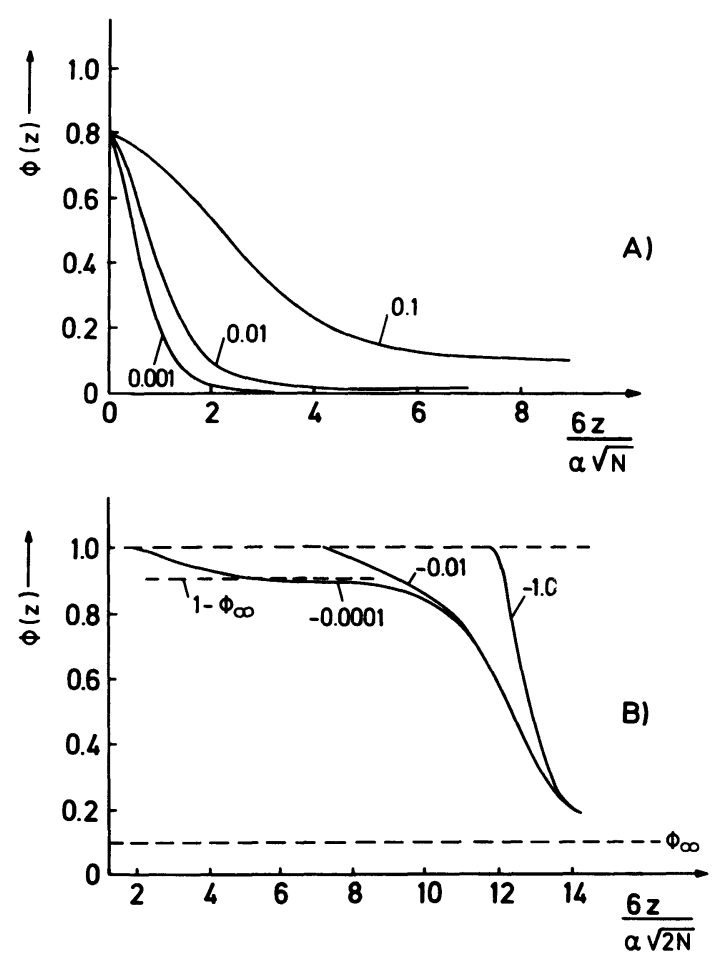

Fig. 1. - Concentration profiles $\Phi(z)$ plotted vs. $z$ at the coexistence curve (A) and in the one phase region (B). Parameter of the curves is $\Phi_{\infty}(\mathrm{A})$ and $N \Delta \mu(\mathrm{B})$. Note that in case (B) the position of the surface is not specified it simply follows from the condition $\Phi(z=0) \equiv \Phi_{1}$.

(for $\Delta \mu=0$ ). As mentioned above, $\Phi_{1}$ only acts as a cut-off on the profile and was arbitrarily chosen $\Phi_{1}=0.8$ in figure $1 \mathrm{~A}$; profiles with smaller (larger) $\Phi_{1}$ are simply found by shifting the ordinate axis to the right (or left, respectively; the missing part of the profile for $\Phi>0.8$ is found from noting that the full interfacial profile is anti-symmetric around the point $\Phi(z)=1 / 2$. This symmetry is no longer true if we allow $\Delta \mu \neq 0$ (Fig. 18). For $\Phi_{1}>1-\Phi_{\infty}$ the surface would be wet for $\Delta \mu=0$ but is coated with a layer of finite thickness only if $\Delta \mu \neq 0$. One can see from figure 1B how the wetting layer develops when $\Delta \mu \rightarrow 0$. Figure 2 shows the growth of the layer thickness $D$ when we approach the wet state along the coexistence curve.

Figure 3 shows our findings on the dependence of the relation between the parameter $g$ and the concentration $\left(\Phi_{\infty}\right)_{\text {tricrit }}$ at which the tricritical point occurs. As emphasized above, for values of $(g)$ of order unity $\left(\Phi_{\infty}\right)_{\text {tricrit }}$ is rather small, particularly for longer chain lenghts. Figure 4 shows then the complete phase diagram of the surface in the $\left(\mu_{1}, \Phi_{\infty}\right)$-plane. For clarity we display rather small values of $N$ and not very large values of $-g$ either, so that the region of first-order wetting can be clearly distinguished. The surface spinodals and the first order line merge tangentially at the tricritical point (one surface spinodal is simply the line $\Phi_{\infty}=1+\mu_{1} / g$ ). In a physically reali-

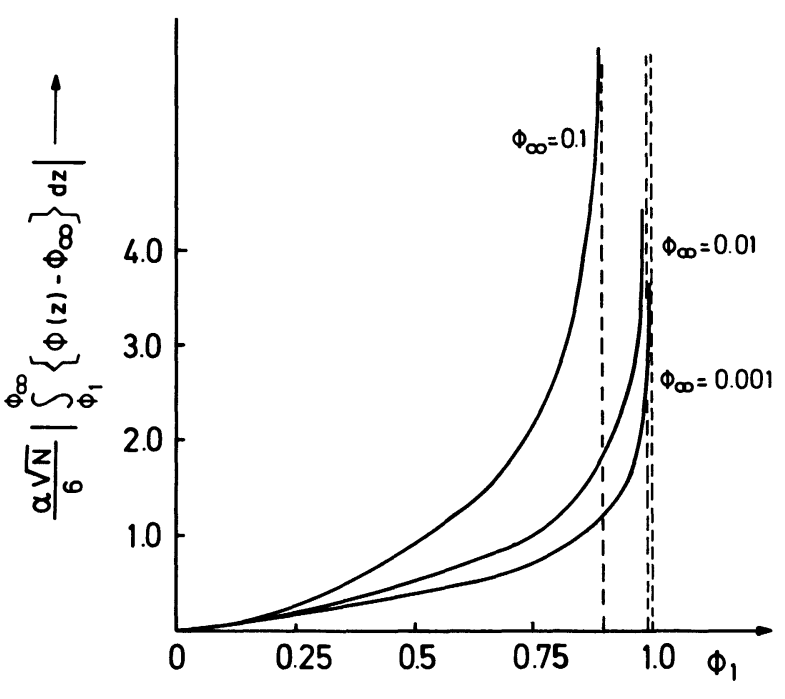

Fig. 2. - Thickness $D$ of the " wet " layer plotted vs. $\Phi_{1}$ for several values of $\Phi_{\infty}$.

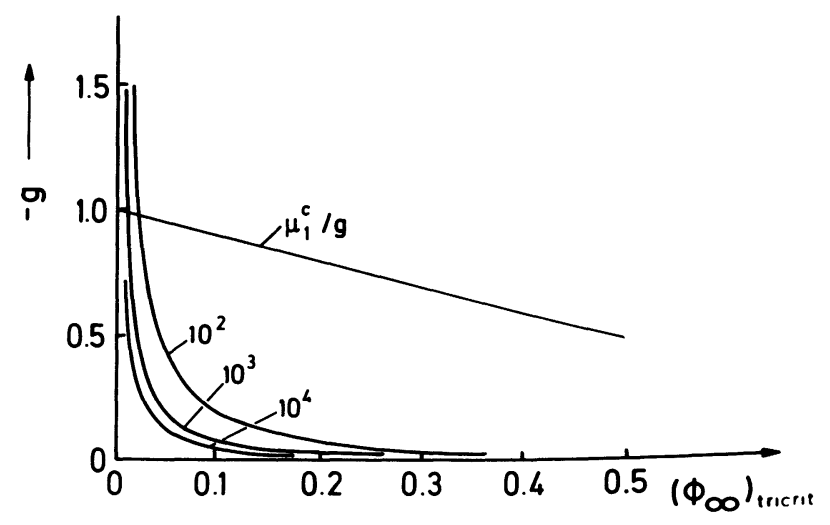

Fig. 3. - Plot of the second derivative of the bare surface free energy $(-g)$ versus the concentration $\left(\Phi_{\infty}\right)_{\text {tricrit }}$ of the wetting tricritical point. Parameter of the curves is $N$. The corresponding normalized first derivative at $\Phi_{1}=0$ at the tricritical point, $\mu_{1}^{\mathrm{c}} / g$, is a straight line independent of $N$ (Eq. (29b)). We choose units where $a=1$.

zable situation, we may change the temperature and thus $\Phi_{\infty}$, but the effective values of $g$ and $\mu_{1}$ then change also (factors of $1 / K_{\mathrm{B}} T$ have been absorbed in their definition, etc.). We hence expect that we cut the phase boundary at the wetting transition by variation of temperature in general under a finite angle. The behaviour then is quantitatively similar to the results observed for a variation of $\mu_{1}$ for $g, \Phi_{\infty}$ held fixed (Figs. 5-8, various examples for other values of the parameters are found in Ref. [51]). We see that at the second-order wetting transition the variation of $\Phi_{1}$ always is rather smooth, while $U_{s}$ has a kink-type maximum there. The susceptibility $\chi_{11}$ is finite at the transition, but exhibits a jump singularity. When $\Phi_{\infty}$ comes close to $\left(\Phi_{\infty}\right)_{\text {tricrit }}$ a rather sharp maximum of $\chi_{11}$ is observed (Fig. 6) which is a precursor of the critical divergence of $\chi_{11}$ found for $\Phi_{\infty}=\left(\Phi_{\infty}\right)_{\text {tricrit }}$. 


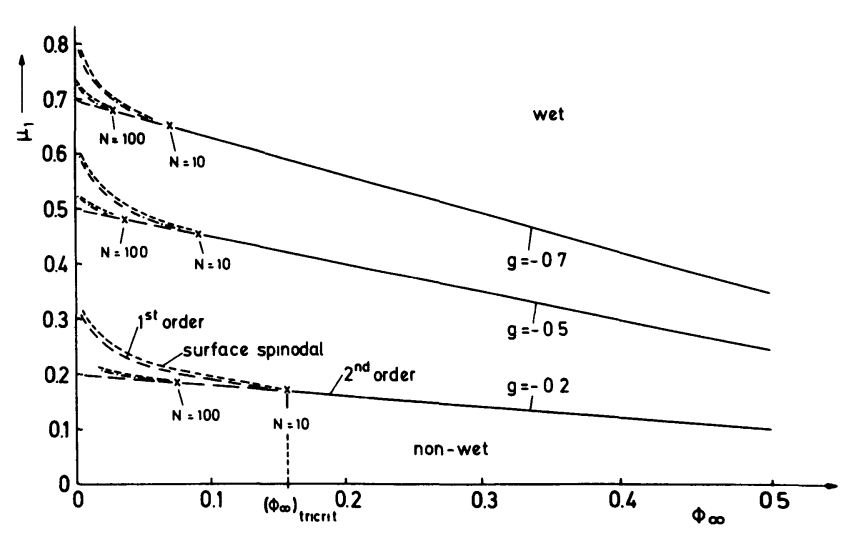

Fig. 4. - Surface phase diagram in the $\left(\mu_{1}, \Phi_{\infty}\right)$ plane for three values of $g$. The region where the surface is wet (at small $\mu_{1}$ ) is separated from the non-wet region by a phase boundary which describes the wetting transition. For $\Phi_{n}>\left(\Phi_{n}\right)_{\text {tricrit }}$ this is just the straight line, equation (29b). The region of first order wetting is shown for both $N=10$, 100 and denoted by dash-dotted curves. In this regime surface spinodals occur and are shown by broken curves.
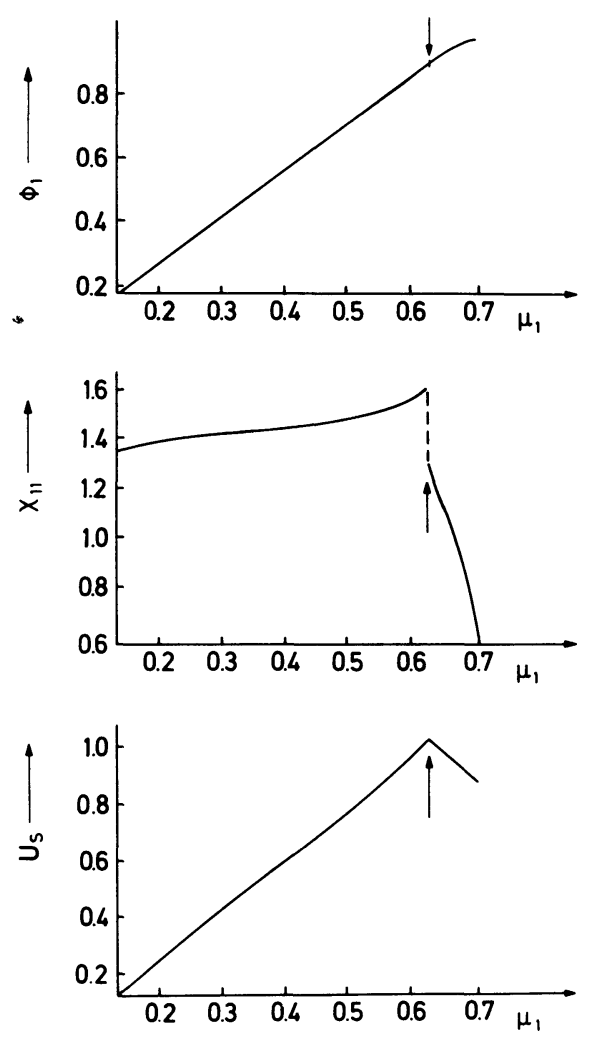

Fig. 5. - Variation of $\Phi_{1}$ (upper part), $\chi_{11}$ (middle part), $U_{\mathrm{s}}$ (lower part) with $\mu_{1}$ for $N=300, \Phi_{\infty}=0.1, g=-0.7$. Arrows show the location of the wetting transition. In this case $\left(\Phi_{\infty}\right)_{\text {tricrit }} \approx 0.017$. Note that $U_{\mathrm{s}}$ as shown here does note contain the factor $\partial \Phi_{\infty} / \partial(1 / T)$ (cf. Eq. (18)).

For $\Phi_{\infty}<\left(\Phi_{\infty}\right)_{\text {tricrit }}$ the wetting transition is weakly first order, and we obtain then jump singularities in $\Phi_{1}$ and $U_{\mathrm{s}}$ as well $\left(\chi_{11}\right.$ then has a delta-function singularity superimposed on its jump). It is seen (Fig. 7)
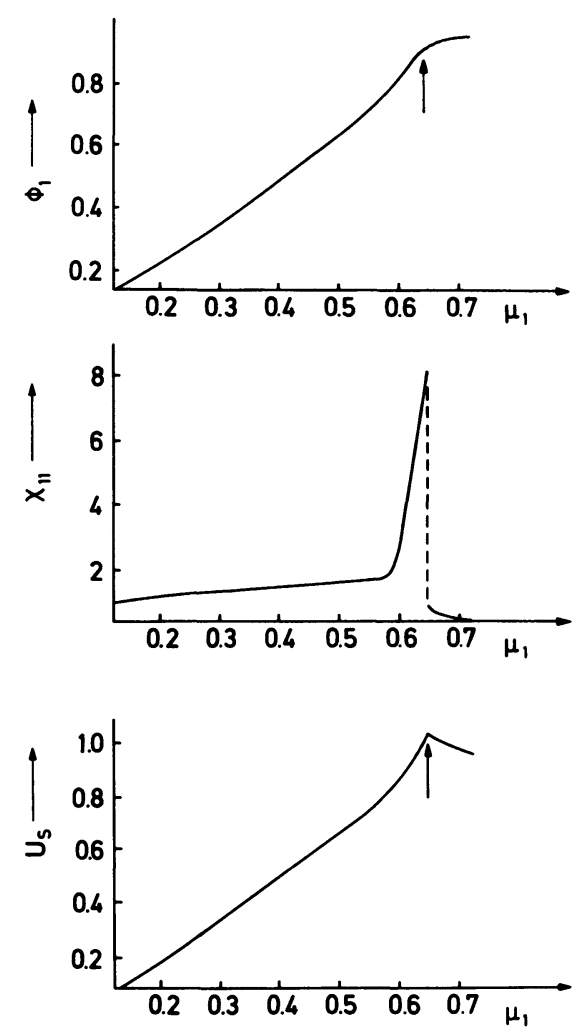

Fig. 6. - Variation of $\Phi_{1}$ (upper part), $\chi_{11}$ (middle part), $U_{\mathrm{s}}$ (lower part) with $\mu_{1}$ for $N=10, \Phi_{\infty}=0.08, g=-0.7$. Arrows show the location of the wetting transition which still is second order but close to the tricritical point $\left(\left(\Phi_{\infty}\right)_{\text {tricrit }} \approx 0.067\right)$.

that the first-order wetting transition occurs at a value $\Phi_{1}^{-}$before $\Phi_{1}$ reaches the value $1-\Phi_{\infty}$ at which critical wetting (Figs. 5,6) would occur, and then $\Phi_{1}$ jumps to a value $\Phi_{1}^{+}$larger than $1-\Phi_{\infty}$. If $N \rightarrow \infty$, however, the fact that $\left(\Phi_{\infty}\right)_{\text {tricrit }} \propto N^{-1 / 2}$ also means that the jump $\Phi_{1}^{+}-\Phi^{-}$vanishes proportional to $N^{-1 / 2}$-already for $N=300$ under otherwise comparable conditions this jump would be hardly visible on the scale of figure 7 , as corresponding calculations show [51].

The behaviour is very different for $g>0$ where critical wetting does not occur and one rather observes always a transition of strongly first order (Fig. 8). Figure 9 shows the phase diagram for a number of typical cases. We note that there exists in this case a particular concentration $\Phi_{\infty}^{*}$ (marked by crosses in Fig. 9). For $\Phi_{\infty}<\Phi_{\infty}^{*}$, we have $\Phi_{1}>\Phi_{\infty}$ in the non wet state and there the profile decreases monotonically, while for $\Phi_{\infty}<\Phi_{\infty}^{*}$ we have $\Phi_{1}<\Phi_{\infty}$ and the profile increases monotonically.

Finally we turn to pre-wetting transitions, which occur for negative $\Delta \mu$ (i.e., in the one-phase region) if the wetting transition for $\Delta \mu=0$ is of first order. The line of these (first-order) pre-wetting transitions ends in a pre-wetting critical point, which again is located analytically from requesting $\chi_{1,1}^{-1}=0$, using equation (15) for $\Delta \mu<0$. The resulting expressions 

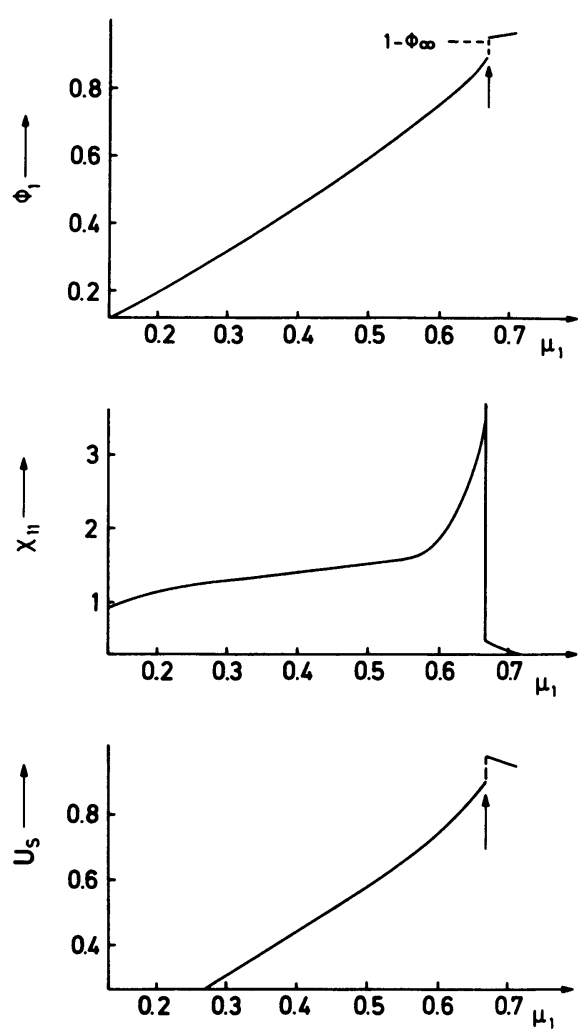

Fig. 7. - Variation of $\Phi_{1}$ (upper part), $\chi_{11}$ (middle part) and $U_{s}$ (lower part) with $\mu_{1}$ for $N=10, \Phi_{\infty}=0.05, g=-0.7$. Arrows show the location of the first-order wetting transition.
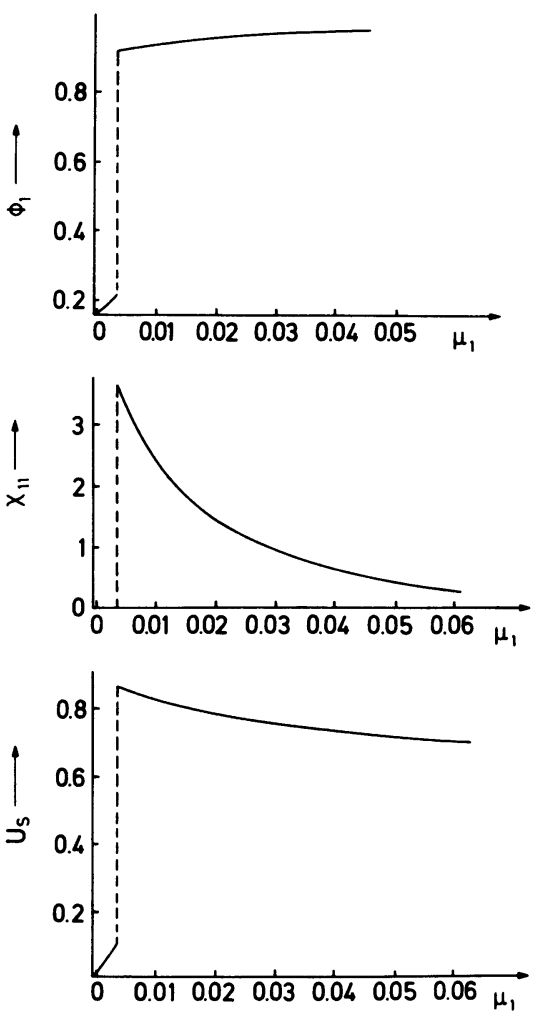

Fig. 8. - Variation of $\Phi_{1}$ (upper part), $\chi_{11}$ (middle part), and $U_{\mathrm{s}}$ (lower part) with $\mu_{1}$ for $N=100, g=0.01$ and $\Phi_{\infty}=0.15$.

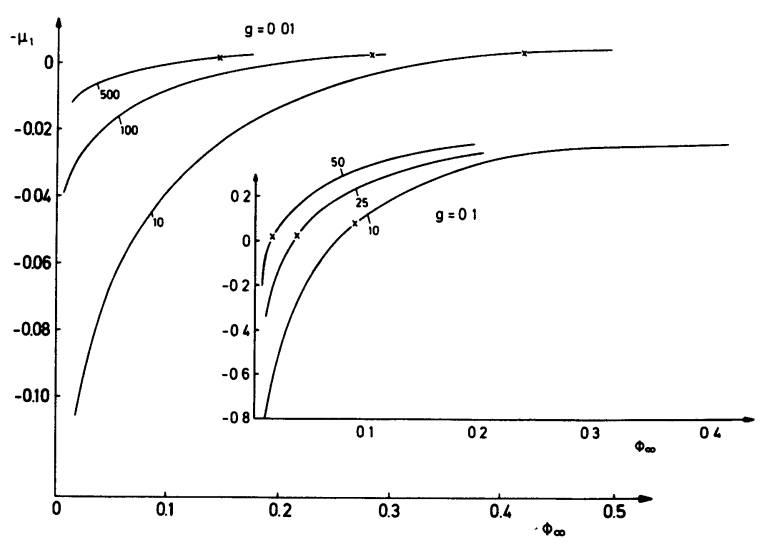

Fig. 9. - Surface phase diagram in the $\left(\mu_{1}, \Phi_{\infty}\right)$ plane for two values of $g>0$ and three values of $N$. The surface is wet below and non-wet above these curves. Crosses mark the point where in the non-wet state the concentration profile is exactly flat, $\mathrm{d} \Phi / \mathrm{d} z=0$.

are straightforward to obtain but lengthy [51] and hence will not be reproduced here. Figure 10 shows, as a typical example, the critical value $g$, as a function of $\Delta \mu$ where the pre-wetting critical point occurs, for typical values of $\Phi_{\infty}$ (which fixes the considered temperature) and chain length.

\section{Fluctuations.}

As always with mean field theories, one has, to worry in which way the statistical fluctuations neglected in this approach may modify the results. This question is particularly relevant for the various second-order transitions possible in the system.

With respect to the bulk critical point of unmixing, fluctuation effects are important only in a very narrow region of $\chi, \Phi_{\infty}$ around the critical point which shrinks to zero as $N \rightarrow \infty[37,40]$; therefore such « bulk » fluctuations need not be considered for the present problem.

With respect to the prewetting critical point, which is an essentially two-dimensional transition in the Ising model universality class [4], the situation already is different. For two spatial dimensions, there is no longer any mean-field regime taking over with increasing chain length $N$, and the behaviour is intrinsically non-classical [37]. As an order parameter of this transition, one may take the jump $\Delta D$ occurring in the thickness $D$ (Eq. (20)) when one crosses the prewetting line defined by $\Delta \mu=\Delta \mu_{\mathrm{c}}\left(\Phi_{\infty}\right)$. The mean field treatment predicts that $D \propto\left[\left(\Phi_{\infty}\right)_{\mathrm{pc}}-\Phi_{\infty}\right]^{1 / 2}$, $\left(\Phi_{\infty}\right)_{\mathrm{pc}}$ being the value of $\Phi_{\infty}$ in the bulk at which temperature the pre-wetting critical point occurs, if one follows the pre-wetting line; in reality we expect that $D \propto\left[\left(\Phi_{\infty}\right)_{\mathrm{pc}}-\Phi_{\infty}\right]^{1 / 8}$, the exponent being that of the two-dimensional Ising model. Also the numerical prediction for $\left(\Phi_{\infty}\right)_{\mathrm{pc}}$ (and $g_{\mathrm{c}}(\Delta \mu)$ as shown in Fig. 10) is quantitatively unreliable, fluctuations tend to shift the critical point to a regime of stronger coupling. 

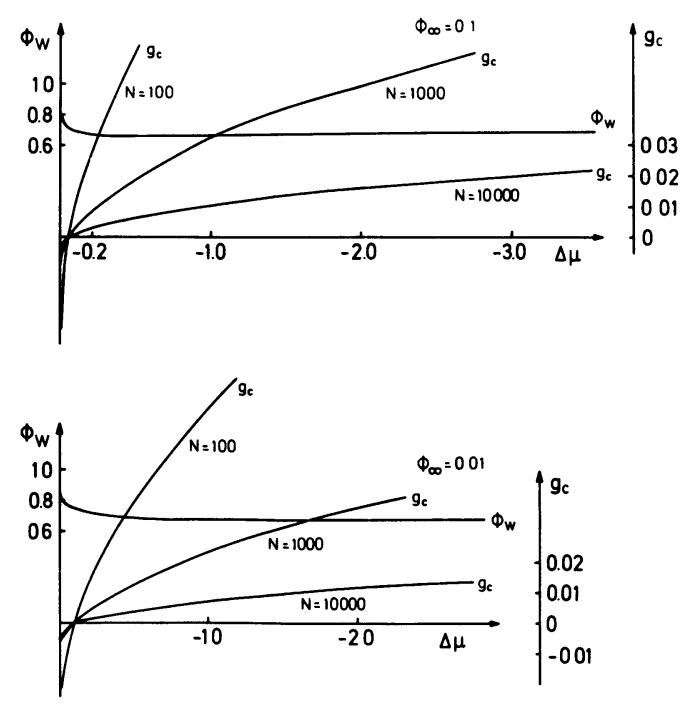

Fig. 10. - Surface phase diagram describing pre-wetting phenomena : in the plane of variables $g, \Delta \mu$ lines $g_{c}(\Delta \mu)$ separate the regime where first-order pre-wetting occurs $\left\{g>g_{\mathrm{c}}(\Delta \mu)\right\}$ from the regime where one smoothly approaches the wet surface when $\Delta \mu \rightarrow 0$. Several choices of $\Phi_{\infty}$ and $N$ are shown. On a separate scale also the value $\Phi_{\mathrm{pw}}$ of the concentration at the pre-wetting critical point is shown.

While these expectations seem plausible on general grounds, we have not made any attempt to study them in any detail.

The situation is more subtle for critical wetting, for which dimensionality $d=3$ is the marginal dimension for the validity of mean field theory [10-12]. While mean field theory predicts that the correlation length $\xi_{\|}$of fluctuations of $\left\langle\Phi_{1}(0) \Phi_{1}(\rho)\right\rangle-\Phi_{1}^{2}$, $\rho$ being a coordinate in the surface plane, diverges with an exponent $v_{\|}=1$ as the transition is approached, this is predicted to be no longer true when fluctuations are taken into account $[10-12,15]$. The relevant fluctuations for this problem are capillary wave excitations of the interface separating the surface layer (whose concentration is $1-\Phi_{\infty}$, at least approximately) from the bulk (which has concentration $\Phi_{\infty}$ ). Taking account of these fluctuations invalidates the concentration profiles as shown in figure 1, of course. In addition, the exponent $v_{\|}$is predicted to be enhanced over its mean field value; taking into account the scaling relation between $v_{\|}$and the exponent $\gamma_{11}$ describing the singularity of $\chi_{11}$ (if there is one) [13],

$$
\gamma_{11}=-2\left(v_{\|}-1\right)
$$

one hence also expects a cusp of $\chi_{11}$ rather than just a finite jump (and hence $\gamma_{11}=0$ ) as in the mean field case (Figs. 5, 6). Note the exponent $v_{\|}$is predicted to be non-universal and to depend on a parameter $w$ defined as

$$
w=\left(4 \pi \sigma \xi_{\text {coex }}^{2}\right)^{-1}
$$

where $\sigma$ is the interfacial free energy between bulk coexisting phases, and $\xi_{\text {coex }}$ the correlation length at the coexistence curve. Near the critical point of a polymer mixture we have [37]

$$
\begin{gathered}
\sigma=\frac{9}{a^{2} \sqrt{N}}\left(1-\frac{\chi_{\text {crit }}}{\chi}\right)^{3 / 2}, \\
\xi_{\text {coex }}=\frac{a}{6} \sqrt{N}\left(\frac{\chi}{\chi_{\text {crit }}}-1\right)^{-1 / 2},
\end{gathered}
$$

where $\chi_{\text {crit }}$ is the value of the Flory-Huggins parameter at criticality. Thus $w$ becomes

$$
w=\left[\pi \sqrt{N}\left(1-\frac{\chi_{\text {crit }}}{\chi}\right)^{1 / 2}\right]^{-1} .
$$

Since the mean field regime of a polymer mixture occurs $[37,40]$ for $\sqrt{N}\left(1-\frac{\chi_{\text {crit }}}{\chi}\right)^{1 / 2} \gg 1$, we hence find that $w \ll 1$; for this regime all theories $[10,11,15]$ agree that $(w<1 / 2)$

$$
v_{\|}=(1-w)^{-1}
$$

and hence

$\gamma_{11}=-2 w /(1-w) \approx-2\left[\pi \sqrt{N}\left(1-\frac{\chi_{\text {crit }}}{\chi}\right)^{1 / 2}\right]^{-1}$.

Thus polymer mixtures offer the interesting possibility to check this prediction of critical wetting theory $[10,11,15]$ by varying the parameter $\sqrt{N}\left(1-\chi_{\text {crit }} / \chi\right)^{1 / 2}$, for instance by changing the temperature and chain length such that one observes critical wetting transitions for different values of the combination $\sqrt{N}\left(1-\chi_{\text {crit }} / \chi\right)^{1 / 2}$. Of course, equations (42, 44) are only valid in the mean field critical region of these mixtures; in the non-mean field critical region very close to criticality, where $\sqrt{N}\left(1-\frac{\chi_{\text {crit }}}{\chi}\right)^{1 / 2} \gtrsim 1$, one crosses over to a value of $w$ which is universal and estimated as $[11,52] w=1.2 \pm 0.3$; the theoretical predictions $[10,11,15]$ still are somewhat controversial for this regime where $w>1 / 2$.

If we go sufficiently away from criticality equations (40) can no longer be used and one crosses over to a regime where the "local " interfacial thickness is no longer given by $\xi_{\text {coex }}$ but rather by $[45-47,49]$ $L \propto a \chi^{-1 / 2}$ (cf. Eq. (34)). Also the interfacial energy $\sigma$ then is $\sigma \propto \chi^{1 / 2} a^{-2}$. We speculate that equation (40) for $w$ should now be replaced by

$$
w=\left(4 \pi \sigma L^{2}\right)^{-1} \propto \chi^{1 / 2} .
$$

Since for mixtures having a critical unmixing point ( $\chi_{\text {crit }}=2 / N$ for symmetric mixtures), we expect that $\chi$ is very small throughout, and hence equation (44) 
would lead to the prediction $\gamma_{11} \propto \chi^{1 / 2}$ which also would be very interesting to test.

As experiments on real materials pertinent to these questions are not yet available, we conclude by briefly describing a few results of a recent Monte Carlo simulation of critical wetting in an Ising model [20,52], figure 11. This model calculation was done with nearest-neighbour exchange only; to model a polymer mixture by an Ising model one would have to choose a large but finite range of the exchange which is controlled by the length of the chains [37]. It is seen that nevertheless the simulation results are remarkably similar to the mean field predictions, figures 5, 6 (of course, there is no obvious way to make the comparison more quantitative, it is not straightforward to translate the parameter of the simulations to those of the mean field calculation). It seems that the critical wetting transition occurs in the simulation at a value $\Phi_{1}$ slightly below $1-\Phi_{\infty}$ already. There occurs a broad finite peak of $\chi_{11}$; probably in this case $w$ is as large as about $w \approx 1.8$,
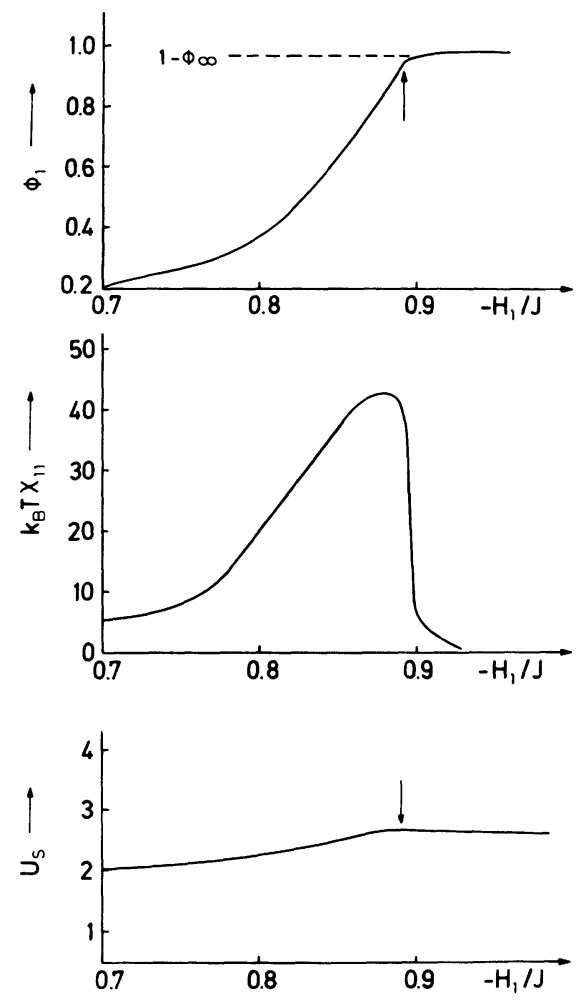

Fig. 11. - Variation of $\Phi_{1}$ (upper part), $\chi_{11}$ middle part) and $U_{s}$ (lower part) with a surface magnetic field $H_{1}$ measured in units of exchange constant $J$ for a simple cubic Ising ferromagnet with a free surface at a temperature $J / K_{\mathrm{B}} T=$ 0.35 (note the critical point occurs at $J / K_{\mathrm{B}} T_{\mathrm{c}} \approx 0.2217$ ) at bulk coexistence (bulk field $H=0$ ). These results are based on Monte Carlo simulations as described in reference [20]. The surface internal energy is measured in units of $J$, and $\chi_{11}$ in this figure is defined as $\chi_{11}=\partial m_{1} / \partial H_{1}, m_{1}$ being the " magnetization " of the first layer (note $\Phi_{1}=$ $\left.\left(1-m_{1}\right) / 2\right)$. and hence a strong divergence of $\xi_{\|}$is predicted $[11,15]$. The associated singularity of $\chi_{11}$ in the Ising model is obviously hard to observe in the simulation, [52]; but then it is likely that it also would be very hard to observe in a real experiment.

We conclude this section by a discussion of effects due to long range surface fields : as mentioned after equation (5), on the basis of van der Waal's forces one would predict that $f_{\mathrm{s}}^{(\mathrm{b})}(\Phi, z) \propto z^{-3}$ for $z \rightarrow \infty$, and then the treatment presented in section 2 also is not quantitatively correct. But it has been shown for mixtures of small molecules and related systems $[17,18]$ that also in this case one may have both firstorder and critical wetting, and hence the possible phase diagrams are qualitatively similar. In this case the marginal dimension for critical wetting is below $d=3$ [13], and hence there is no need to worry about the above fluctuation effects. Since our model calculations anyway are not a faithful representation of a real polymeric mixture even in the bulk (there are effects of asymmetry, chain polydispersity, unknown dependence of $\chi$ on volume fraction, temperature, and even chain length), we have not undertaken the large numerical effort to perform explicit calculations for this case as well.

\section{Conclusions.}

In this paper, we have given a mean field analysis of wetting transitions of polymer mixtures. Even on the mean field level, our results are still somewhat qualitative because of (i) our use of the long-wavelength approximation and (ii) restriction to the case of short range surface perturbations. In spite of these restrictions, we believe that the following conclusions are of general validity :

a) for physically reasonable regimes of parameters, second-order wetting transitions can be found. It would be interesting to check this prediction experimentally. If one could realize a situation where the short-range part of the surface forces is much larger than the long range van der Waals's part, one would have an approximate realization of a system with fluctuation corrections to mean field but the constant $w$ (Eqs. (40), (42)) being very small, which is very interesting because of the predicted non-universal critical behaviour;

b) physically the forces exerted by the wall to the mixture are fixed and can not easily be varied. In polymer mixtures one can vary the chain lengths $N_{\mathrm{A}}, N_{\mathrm{B}}$ of the constituents, and due to the existence of a second disposable parameter (in addition to temperature) one has more experimental freedom in studying wetting transitions, than in other mixtures;

c) since one expects for partially miscible polymer mixtures the unmixing tendency at the surface to be relatively strong in comparison to that of the bulk, 
a rather strong surface enrichment of one constituent of the mixture must be expected even away from two-phase coexistence. This phenomenon may become important particularly for applications of thin films of polymer blends.

\section{Acknowledgments.}

One of us has benefitted from stimulating discussions with D. M. Kroll, P. A. Pincus and M. Schick. This research is supported in part by Sonderforschungsbereich 41 der Deutschen Forschungsgemeinschaft.

\section{References}

[1] Cahn, J. W., J. Chem. Phys. 66 (1977) 3667 ; see also Ebner, C. and SAAM, W. F., Phys. Rev. Lett. 38 (1977) 1486 ;

SaAm, W. F. and Ebner, C., Phys. Rev. A 17 (1983) 1768.

[2] Pandit, R. and Wortis, M., Phys. Rev. B 25(1982) 3226.

[3] Pandit, R., Schick, M. and Wortis, M., Phys. Rev. $B 26$ (1982) 5112.

[4] Nakanishi, H. and Fisher, M. E., Phys. Rev. Lett. 49 (1982) 1565.

[5] Sullivan, D. W., Phys. Rev. B 20 (1979) 3991, J. Chem. Phys. 74 (1981) 2604.

[6] Hauge, E. H. and Schick, M., Phys. Rev. B 27 (1983) 4288.

[7] Tarazona, P. and Evans, R., Mol. Phys. 48 (1983) 799 ; Evans, R. and Taranzona, P., J. Chem. Phys. 80 (1984) 587.

[8] Nightingale, M. P., SaAm, W. F. and Schick, M., Phys. Rev. Lett. 51 (1983) 1275.

[9] Kroll, D. M. and Lipowsky, R., Phys. Rev. B 26 (1982) 5289.

[10] Lipowsky, R., Kroll, D. M. and ZiA, R. K. P., Phys. Rev. B 27 (1983) 4499.

[11] Brézin, E., HalPerin, B. I. and Leibler, S., Phys. Rev. Lett. 50 (1983) 1387.

[12] Brézin, E., Halperin, B. I. and Leibler, S., J. Physique 44 (1983) 775.

[13] LiPowsky, R., Phys. Rev. Lett. 52 (1984) 1429 ; Z. Phys. B 55 (1984) 345 ; J. Appl. Phys. 55 (1984) 2485.

[14] Lipowsky, R. and Kroll, D. M., Phys. Rev. Lett. 52 (1984) 2303.

[15] Fisher, D. S. and HuSE, D. A., preprint.

[16] Kroll, D. M. and Meister, T. F., Phys. Rev. B 31 (1985) 392.

[17] Dietrich, S. and Schick, M., Phys. Rev. B 31 (1985) 4718.

[18] Ebner, C., SAaM, W. F. and Sen, A. K., preprint.

[19] LuCK, J. M., LeIBleR, S. and DeRridA, B., preprint ; Nightingale, M. P. and InDekeU, J. O., preprint.

[20] Binder, K. and LANDaU, D. P., J. Appl. Phys. 57 (1985) 3306.

[21] Forgacs, G., OrLand, H. and Schick, M., preprint.

[22] Forgacs, G., OrLand, H. and Schick, M. preprint.

[23] Nakanishi, H. and Pincus, P., J. Chem. Phys. 79 (1983) 997.

[24] Recent reviews are Sullivan, D. E. and Telo da Gama, M. M., Fluid Interfacial Phenomena C. A. Croxton, Ed. (Wiley, New York) 1985, and HaUge E. M., Fundamental Problems in Statistical Mechanics VI E. G. D. Cohen, Ed. North Holland, Amsterdam) 1985.

[25] Moldover, M. R. and CAHN, J. W., Science 207 (1980) 1073.
[26] Kwon, O. D., Beaglehole, D., Webb, W. W., Widom, B., SCHMidT, J. W., CAHN, J. W., Moldover, M. R. and Stephenson, R., Phys. Rev. Lett. 48 (1982) 185.

[27] Pohl, D. W. and Goldburg, W. I., Phys. Lett. 48 (1982) 111.

[28] Schmidt, J. W. and Moldover, M. R., J. Chem. Phys. 79 (1983) 379.

[29] Mochrie, S. G. J., Sutton, M., Birgeneau, R. J., Morcton, D. E. and Horn, P. M., Phys. Rev. B 30 (1984) 263.

[30] Awschalom, D. D., Lewis, G. N. and Gregory, S., Phys. Rev. Lett. 51 (1983) 581.

[31] Hamilton, J. J. and Goodstein, D. L., Phys. Rev. B 28 (1983) 3838

[32] Krim, J., Dash, J. G. and Suzanne, J., Phys. Rev. Lett. 52 (1984) 640 .

[33] Suzanne, J., Sequin, J. L., Bienfait, M. and Lerner, E., Phys. Rev. Lett. 52 (1984) 637.

[34] Halary, J. L., Ubrich, J. M., NunZI, J. M., MONNERIE, L. and SteIn, R. S., Polymer 25 (1984) 956 ;

UBRICH, J. M., Thesis (ESPCI, Paris 1984, unpublished).

[35] FlORY, P. J., Principles of Polymer Chemistry (Cornell University Press, Ithaca) 1971.

[36] De Gennes, P. G., J. Chem. Phys. 72 (1980) 4756; Pincus, P., J. Chem. Phys. 75 (1981) 1996.

[37] Binder, K., J. Chem. Phys. 79 (1983) 6387; Phys. Rev. A 29 (1984) 341.

[38] Nishi, T., WANG, T. T. and KweI, T. K., Macromolecules 8 (1975) 277 ;

Gelles, R. and FranK, C. W., Macromolecules 15 (1982) 486 ;

SNYder, H. L., Meakin, P. and Reich, S., Macromolecules 16 (1983) 757;

Snyder, H. L. and Meakin, P. A., J. Chem. Phys. 79 (1983) 5588 ;

Torkelson, J. M., Pierell, M. and Frank, C. W., Macromolecules 17 (1984) 1505;

Hashimoto, T., Kumaki, J. and KawaI, H., Macromolecules 16 (1983) 641

[39] For reviews see Gunton, J. D., SAN Miguel, M. and SAHNI, P. S., Phase Transitions and Critical Phenomena C. Domb and J. L. Lebowitz, Eds. (Academic Press, New York) 8 1983, p. 267;

BiNDER, K., Condensed Matter Research Using Neutrons S. W. Lovesey and R. Scherm, Eds. (Plenum Press, New York) 1984.

[40] DE Gennes, P. G., J. Phys. Lett. 38 (1977) L-441; JoANNy, J. F., J. Phys. A 11 (1978) L117.

[41] More details are found in reference [20] and in BiNDER, K., LANDAU, D. P. and Kroll, D. M., to be published. 
[42] Koningsveld, R., KleintJens, L. A. and SchoffeleERS, H. M., Pure Appl. Chem. 39 (1974) 1.

[43] MC MASTER, L. P., Macromolecules 6 (1973) 760 ; FLORY, P. J., J. Am. Chem. Soc. 87 (1965) 1833 ;

Flory, P. J., ORWOll, R. A. and VRIJ, A., J. Am. Chem. Soc. 86 (1964) 3515.

[44] DE Gennes, P. G., Scaling Concepts in Polymer Physics (Cornell University Press, Ithaca) 1979.

[45] BINDER, K. and FRISCH, H. L., Macromolecules 17 2928 (1984).

[46] Helfand, E. and Tagami, Y., J. Polym. Sci. B 9 (1971) 741 ; J. Chem. Phys. 56 (1971) 3592; ibid. 57 (1971) 1812.

[47] Helfand, E., J. Chem. Phys. 62 (1975) 999 ; 63 (1975) 2192.

Helfand, E. and SAPSe, A. M., J. Chem. Phys. 62 (1975) 1327 ;

Helfand, E. and Weber, T. A., Macromolecules 9 (1976) 311 ;
Helfand, E., Mácromolecules 9 (1976) 307 ; Acc. Chem. Res. 8 (1975) 295.

[48] Joanny, J. F. and Leibler, L., J. Physique 39 (1978) 951.

[49] Noolandi, J. and Hong, K. M., Macromolecules 15 (1982) 482 ;

HoNG, K. M. and Noolandi, J., Macromolecules 14 (1981) 727 ; 16 (1983) 1083.

[50] We here follow the notation commonly used in the context of surface critical phenomena; see e.g. BINDER, K., in Phase Transitions and Critical Phenomena C. Domb and J. L. Lebowitz, Eds. (Academic Press, New York) 8 (1983) p. 1.

[51] Schmid,, I., Dissertation (Johannes Gutenberg Universität Mainz) in preparation.

[52] Binder, K., Landau, D. P. and Kroll, D. M., in preparation. 